\title{
A Derivation of Free-rotation in the Three-dimensional Space
}

\author{
Legend Chen ${ }^{1}$ \\ ${ }^{1}$ University of Shanghai for Science and Technology optoelectronic information and \\ Computer Engineering Shanghai 200093 China
}

\begin{abstract}
The paper describes an algorithm derivation of rotation in the three-dimensional space, which is an efficient method for rotating objects in the space. The key in the derivation process is to summarize the many thoughts on this issue, included the geometric algebra, coordinate transformation, linear space and matrix operations, and analysis of some possible algorithm of rotation, such as basic rotation, the combination of rotations and coordinates system transformation. Finally, obtains a matrix as a formula for the free-rotation in the three dimensions.
\end{abstract}

Keywords: Three-dimensional, Freerotation, Computer Graphics, Coordinate Transformation

The rotation in two-dimensions is easy to draw, but also well understood. However, How it be extended to the freerotation in the three-dimensions?

\section{Basic Rotation in a Plane}

Using triangle transformation formula, you can get the following formula for a rotating basis.

$$
\begin{aligned}
& X \cdot \cos \theta-Y \cdot \sin \theta=X^{\prime} \\
& X \cdot \sin \theta+Y \cdot \cos \theta=Y^{\prime}
\end{aligned}
$$

The formula is easy to conclude from the triangle transformation by the following equation.

$$
\begin{array}{ll}
r \cdot \cos (\theta+\alpha)=X^{\prime} & \mathrm{r} \cdot \cos \alpha=X \\
r \cdot \sin (\theta+\alpha)=Y^{\prime} & , \quad \mathrm{r} \cdot \sin \alpha=Y
\end{array}
$$

And

$$
\begin{aligned}
& \mathrm{r} \cdot \cos \alpha \cdot \cos \theta-\mathrm{r} \cdot \sin \alpha \cdot \sin \theta=X^{\prime} \\
& \mathrm{r} \cdot \cos \alpha \cdot \sin \theta+\mathrm{r} \cdot \sin \alpha \cdot \cos \theta=Y^{\prime}
\end{aligned}
$$

$\theta$ is the angle of rotation, and $\alpha$ is the angle of vector.

This formula is a very classical method of rotation in the two-dimensional space. Parameters are the angle and vector, return a coordinate vector, very concise.

\section{Continue to Rotate in the Space}

In three dimensions space, using the known formula previously mentioned, corresponding to calculation on the $\mathrm{YZ}$ plane, $X Z$ plane and $X Y$ plane, the rotation around the $\mathrm{X}$ axis, $\mathrm{Y}$ axis and $\mathrm{Z}$ axis can be resolved directly.

Thus, there is a complex method to complete a free rotation with a series of free rotation, which seems feasible. After all, we just want to find a plane can be rotated.

Based on the main idea of the previous section, the algorithm is as follows:

Defines a vector $\mathrm{P}$, that the location and direction of rotation axis. 
- Step 1: Translate P, so that the starting point of $\mathrm{P}$ coincides with the coordinate origin

- Step 2: Rotate P around the X axis, which another point of $\mathrm{P}$ is to the $\mathrm{XZ}$ plane

- Step 3: Rotate P around the Y axis, which another point of $\mathrm{P}$ coincides with the $\mathrm{Z}$ axis

- Step 4: Rotate $\mathrm{P}$ around the $\mathrm{Z}$ axis, which

- Step 5: Y axis reverse, inverse transformation

- Step 6: X axis reverse

- Step 7: Translation reverse

This is definitely a product of a lot of triangle rotations and translations. It is undeniable that there are too many variables to calculate the intermediate. What's worse, no efficiencies, the accuracy is also unknown (be mentioned later and give a proof).

\section{Gain a New Coordinate System}

Following the above ideas, the new problem lies in the coordinate system transformation. We need to create a new coordinate system based on one shaft, and get new space coordinate information.

Imitation of pinhole imaging, just a way, can be used to simulate the new coordinate system. Of course, you could also simulate a projector, but soon you will find that it is merely a geometric algebra approach and a quite timeconsuming processing.

Based on the geometric algebra, the approach is as follows:

- Step 1: Using operation of dot product and cross product with right-hand rule, obtain three orthogonal vectors presenting new coordinates.

$$
\begin{aligned}
& v 1 \\
& v 2^{\prime}=v-v \cdot \frac{v 1 g v 2}{|v 1||v 2|} \\
& v 3=v 1 \times v 2
\end{aligned}
$$

$\mathrm{v} 2$ is random vector, not parallel to $\mathrm{v} 1$. Convert them to unit vectors. Then, the coordinates consist of unit vectors of $\mathrm{v} 1$, v2' and v3.

- Step 2: Comparison of the point in the space with the coordinates, calculate their relative displacement.

Defines vectors of v1, v2, v3 and v0 in the new coordinates, that $\mathrm{v} 1$ as a new $\mathrm{X}$ axis, v2 as a new $\mathrm{Y}$ axis, v3 as a new $\mathrm{Z}$ axis and $\mathrm{v} 0$ as a relative position.

$$
\begin{gathered}
v 0^{\prime}=v 0-\left(v 0 \cdot \frac{v 0^{\prime} \mathrm{g} v 3}{\left|v 0^{\prime} \| v 3\right|}\right) \\
\cos \theta=\frac{v 0^{\prime} \mathrm{g} v 1}{\left|v 0^{\prime} \| v 1\right|} \\
\sin \theta=\frac{v 0^{\prime} \mathrm{g} v 2}{\left|v 0^{\prime} \| v 2\right|}
\end{gathered}
$$

$|v 1|,|v 2|,|v 3|$ value is 1 . The $r$ is the distance between the point with the new origin.

Results

$$
\begin{aligned}
& x^{\prime}=r \cdot \cos \theta \\
& y^{\prime}=r \cdot \sin \theta \\
& z^{\prime}=\frac{v 3 g v 0}{|v 3|}
\end{aligned}
$$

It is easy to understand, but the inverse transformation is impossible in the rotation transformation. Its calculation still spends so much, which not will be used in the practical application. 


\section{Transformation of Coordinate Sys- tem}

Now we come to the point. In order to express clearly, I use linear algebra through the matrix to solve the problem of coordinate transformation,

Simply, skip shift thing. In other word, the new coordinate system origin is the origin of the old coordinate system.

The following matrix presents a normal coordinate system.

$$
\left(\begin{array}{lll}
1 & 0 & 0 \\
0 & 1 & 0 \\
0 & 0 & 1
\end{array}\right)
$$

As long as it is an orthogonal matrix, each column vector can be regarded as a sub-vector of a coordinate axis. They are linearly independent, orthogonal.

$$
\left(\begin{array}{lll}
1 & 0 & 0 \\
0 & 1 & 0 \\
0 & 0 & 1
\end{array}\right)\left(\begin{array}{l}
a \\
b \\
c
\end{array}\right)=a\left(\begin{array}{l}
1 \\
0 \\
0
\end{array}\right)+b\left(\begin{array}{l}
0 \\
1 \\
0
\end{array}\right)+c\left(\begin{array}{l}
0 \\
0 \\
1
\end{array}\right)=\left(\begin{array}{l}
a \\
b \\
c
\end{array}\right)
$$

For a space vector, "a" is the value of $\mathrm{X}$ axis component, " $\mathrm{b}$ " is the value of $\mathrm{Y}$ axis component, " $\mathrm{c}$ " is the value of $\mathrm{Z}$ ax is component, and the combination of these components is the vector itself.

Suppose there is a point in a new coordinate system corresponds to the other point in the space coordinate system.

$$
\left(\begin{array}{lll}
a_{11} & a_{12} & a_{13} \\
a_{21} & a_{22} & a_{23} \\
a_{31} & a_{32} & a_{33}
\end{array}\right)\left(\begin{array}{l}
x \\
y \\
z
\end{array}\right)=\left(\begin{array}{lll}
1 & 0 & 0 \\
0 & 1 & 0 \\
0 & 0 & 1
\end{array}\right)\left(\begin{array}{l}
a \\
b \\
c
\end{array}\right)
$$

Because it is orthogonal matrix, the following formula established

$$
A A^{-1}=A A^{T}=E
$$

Then

$$
\left(\begin{array}{l}
x \\
y \\
z
\end{array}\right)=\left(\begin{array}{lll}
a_{11} & a_{12} & a_{13} \\
a_{21} & a_{22} & a_{23} \\
a_{31} & a_{32} & a_{33}
\end{array}\right)^{-1}\left(\begin{array}{l}
a \\
b \\
c
\end{array}\right)=\left(\begin{array}{lll}
a_{11} & a_{12} & a_{13} \\
a_{21} & a_{22} & a_{23} \\
a_{31} & a_{32} & a_{33}
\end{array}\right)^{T}\left(\begin{array}{l}
a \\
b \\
c
\end{array}\right)
$$

Considering the case of translation, the formula can be expressed in a different sense, as the following two.

$$
\begin{aligned}
& \left.\left(\begin{array}{l}
x \\
y \\
z
\end{array}\right)=\left(\begin{array}{lll}
a_{11} & a_{12} & a_{13} \\
a_{21} & a_{22} & a_{23} \\
a_{31} & a_{32} & a_{33}
\end{array}\right)^{T}\left(\begin{array}{l}
a \\
b \\
c
\end{array}\right)-\left(\begin{array}{l}
u \\
v \\
w
\end{array}\right)\right) \\
& \left(\begin{array}{l}
a \\
b \\
c
\end{array}\right)=\left(\begin{array}{lll}
a_{11} & a_{12} & a_{13} \\
a_{21} & a_{22} & a_{23} \\
a_{31} & a_{32} & a_{33}
\end{array}\right)\left(\begin{array}{l}
x \\
y \\
z
\end{array}\right)+\left(\begin{array}{l}
u \\
v \\
w
\end{array}\right)
\end{aligned}
$$

Note: $(\mathrm{u}, \mathrm{v}, \mathrm{w})$ as a translation vector

\section{Rotation in a New Coordinate Sys- tem}

According to the rotation axis coincides with the new $\mathrm{Z}$ axis, we create a new coordinate system (presented as a matrix A if it's exited, which certainly exited), rotate it, and then converted back to the original coordinates.

Here is an example.

Equation of plane rotation

$$
\left(\begin{array}{l}
x^{\prime \prime} \\
y^{\prime \prime} \\
z^{\prime \prime}
\end{array}\right)=\left(\begin{array}{ccc}
\cos \theta & -\sin \theta & 0 \\
\sin \theta & \cos \theta & 0 \\
0 & 0 & 1
\end{array}\right)\left(\begin{array}{l}
x^{\prime} \\
y^{\prime} \\
z^{\prime}
\end{array}\right)
$$

There is a $\mathrm{z}$-axis rotation matrix, and $\theta$ is the angle of rotation.

To apply the two formulas derived previously (1), (2)

$$
\left(\begin{array}{l}
x \\
y \\
z
\end{array}\right)=A\left(\begin{array}{l}
x^{\prime \prime} \\
y^{\prime \prime} \\
z^{\prime \prime}
\end{array}\right)+\left(\begin{array}{l}
u \\
v \\
w
\end{array}\right),\left(\begin{array}{l}
x^{\prime} \\
y^{\prime} \\
z^{\prime}
\end{array}\right)=A^{T}\left(\begin{array}{l}
a \\
b \\
c
\end{array}\right)-\left(\begin{array}{l}
u \\
v \\
w
\end{array}\right)
$$


( $a, b, c)$ as the initial point. ( $x^{\prime}, y^{\prime}, z^{\prime}$ ) as a the point in new coordinate system. $(\mathrm{x}, \mathrm{y}, \mathrm{z})$ as a point after a rotation. ( $\mathrm{x}, \mathrm{y}$, $\mathrm{z}$ ) as a result point.

$$
\left(\begin{array}{l}
x \\
y \\
z
\end{array}\right)=A\left(\begin{array}{ccc}
\cos \theta & -\sin \theta & 0 \\
\sin \theta & \cos \theta & 0 \\
0 & 0 & 1
\end{array}\right) A^{T}\left(\left(\begin{array}{l}
a \\
b \\
c
\end{array}\right)-\left(\begin{array}{l}
u \\
v \\
w
\end{array}\right)\right)+\left(\begin{array}{l}
u \\
v \\
w
\end{array}\right)
$$

The previously mentioned method with a series of axis rotating, it is also obvious correctness, which are all orthogonal transformations.

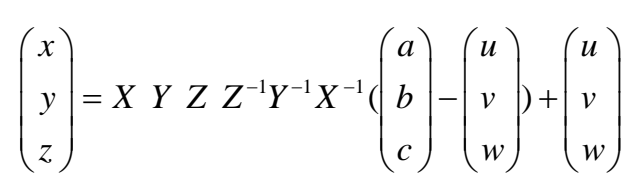

\section{Optimization for the Algorithm}

Coordinate transformation method can be used to get answers to the above issue of this free rotation. However, in the above mentioned transformation matrix, the first column and second column vectors are no other special requirements, just compliance with the orthogonal condition and unit condition.

Of course, the frame of reference really has nothing to do with the rotation. By calculating, we should be able to replace the intermediate variables to achieve the purpose of simplifying the formula equation.

As a coordinate system transformation matrix

$$
A=\left(\begin{array}{lll}
a 1 & b 1 & c 1 \\
a 2 & b 2 & c 2 \\
a 3 & b 3 & c 3
\end{array}\right)
$$

Then, the rotate equation $\left(\begin{array}{lll}r 11 & r 12 & r 13 \\ r 21 & r 22 & r 23 \\ r 31 & r 32 & r 33\end{array}\right)=A\left(\begin{array}{ccc}\cos \theta & -\sin \theta & 0 \\ \sin \theta & \cos \theta & 0 \\ 0 & 0 & 1\end{array}\right) A^{T}$

In accordance with the conditions of the orthogonal relationship, their dot product and cross product is zero.

$$
\begin{aligned}
& a 1 \cdot b 1+a 2 \cdot b 2+a 3 \cdot b 3=0 \\
& c 1 \cdot b 1+c 2 \cdot b 2+c 3 \cdot b 3=0 \\
& a 1 \cdot c 1+a 2 \cdot c 2+a 3 \cdot c 3=0 \\
& a 1 \cdot a 1+a 2 \cdot a 2+a 3 \cdot a 3=1 \\
& b 1 \cdot b 1+b 2 \cdot b 2+b 3 \cdot b 3=1 \\
& c 1 \cdot c 1+c 2 \cdot c 2+c 3 \cdot c 3=1
\end{aligned}
$$

Addition of the right-hand rule

$$
\begin{aligned}
& a 2 \cdot b 3-a 3 \cdot b 2=c 1 \\
& a 3 \cdot b 1-a 1 \cdot b 3=c 2 \\
& a 1 \cdot b 2-a 2 \cdot b 1=c 3
\end{aligned}
$$

r11:

$\mathrm{c} \cdot \mathrm{a} 1 \cdot \mathrm{a} 1+\mathrm{s} \cdot \mathrm{a} 1 \cdot \mathrm{b} 1-\mathrm{s} \cdot \mathrm{a} 1 \cdot \mathrm{b} 1+\mathrm{c} \cdot \mathrm{b} 1 \cdot \mathrm{b} 1+\mathrm{c} 1 \cdot \mathrm{c} 1$ $=(1-\mathrm{c}) \cdot \mathrm{c} 1 \cdot \mathrm{c} 1+\mathrm{c}$

r21 :

$\mathrm{c} \cdot \mathrm{a} 1 \cdot \mathrm{a} 2+\mathrm{s} \cdot \mathrm{a} 1 \cdot \mathrm{b} 2-\mathrm{s} \cdot \mathrm{a} 2 \cdot \mathrm{b} 1+\mathrm{c} \cdot \mathrm{b} 1 \cdot \mathrm{b} 2+\mathrm{c} 1 \cdot \mathrm{c} 2$

$=\mathrm{s} \cdot \mathrm{c} 3+(1-\mathrm{c}) \cdot \mathrm{c} 1 \cdot \mathrm{c} 2$

r31:

$\mathrm{c} \cdot \mathrm{a} 1 \cdot \mathrm{a} 3+\mathrm{s} \cdot \mathrm{a} 1 \cdot \mathrm{b} 3-\mathrm{s} \cdot \mathrm{a} 3 \cdot \mathrm{b} 1+\mathrm{c} \cdot \mathrm{b} 1 \cdot \mathrm{b} 3+\mathrm{c} 1 \cdot \mathrm{c} 3$ $=-\mathrm{s} \cdot \mathrm{c} 2+(1-\mathrm{c}) \cdot \mathrm{c} 1 \cdot \mathrm{c} 3$

Therefore, the final result of the transformation matrix can be calculated as (3). Its parameter is only a " $\mathrm{c}$ " vector, the direction vector of the rotation axis. 


$$
\left(\begin{array}{ccc}
(1-\cos \theta) \cdot c 1^{2}+\cos \theta & -\sin \theta \cdot c 3+(1-\cos \theta) \cdot c 1 \cdot c 2 & \sin \theta \cdot c 2+(1-\cos \theta) \cdot c 1 \cdot c 3 \\
\sin \theta \cdot c 3+(1-\cos \theta) \cdot c 1 \cdot c 2 & (1-\cos \theta) \cdot c 2^{2}+\cos \theta & -\sin \theta \cdot c 1+(1-\cos \theta) \cdot c 2+c 3 \\
-\sin \theta \cdot c 2+(1-\cos \theta) \cdot c 1 \cdot c 3 & \sin \theta \cdot c 1+(1-\cos \theta) \cdot c 2+c 3 & (1-\cos \theta) \cdot c 3^{2}+\cos \theta
\end{array}\right)
$$

\section{Conclusions}

The final formula for the free-rotation in the three dimensions is Simple and easy to use. The thinking of the derivation plays a significant role in the whole transformation process. Reference frame transformation has been applied to solve many complex problems such as $\mathrm{z}$ transform, w' transform.

\section{References}

[1] G. E. Shilov, "Linear Algebra," Prentice Hall, 1971.

[2] D. F. Rogers, "Procedural Elements for Computer Graphics," China Machine Press, 2002.

[3] J. W. Brown, and R. V. Churchill, "Complex Variables and Application," China Machine Press, 2004.

[4] M. Gross, and H. Pfister, "Pointbased graphics," Elsevier, 2007 